\title{
Frontières
}

\section{Policières en marge de la « vraie police »}

\section{Line Beauchesne}

Volume 14, numéro 1, automne 2001

\section{Où est la marge?}

URI : https://id.erudit.org/iderudit/1074158ar

DOI : https://doi.org/10.7202/1074158ar

Aller au sommaire du numéro

Éditeur(s)

Université du Québec à Montréal

ISSN

1180-3479 (imprimé)

1916-0976 (numérique)

Découvrir la revue

\section{Citer cet article}

Beauchesne, L. (2001). Policières en marge de la « vraie police ». Frontières, 14(1), 43-48. https://doi.org/10.7202/1074158ar

\section{Résumé de l'article}

Lorsqu'une profession est typée masculine, cela signifie que la pratique même du métier est une preuve de masculinité. C'est le cas du métier de policier dans ses fonctions de répression. Le vocabulaire du milieu de travail en témoigne. $\mathrm{Au}$ bas de l'échelle, il y a tout le travail de bureau et de services à la communauté, perçu comme étant plus féminin, et au sommet il y a le travail lié à la répression des activités criminelles, là où l'on fait de la "vraie " police (Hunt, 1990). Car ce ne sont pas toutes les tâches dans la police qui sont perçues comme du vrai travail de policier, c'est-à-dire comme des lieux d'affirmation de cette masculinité 


\section{Résumé}

Lorsqu'une profession est typée masculine, cela signifie que la pratique même du métier est une preuve de masculinité. C'est le cas du métier de policier dans ses fonctions de répression. Le vocabulaire du milieu de travail en témoigne. Au bas de l'échelle, il y a tout le travail de bureau et de services à la communauté, perçu comme étant plus féminin, et au sommet il y a le travail lié à la répression des activités criminelles, là où l'on fait de la "vraie » police (Hunt, 1990). Car ce ne sont pas toutes les tâches dans la police qui sont perçues comme du vrai travail de policier, c'est-à-dire comme des lieux d'affirmation de cette masculinité.

Mots clés : police - discrimination sexisme

\section{Abstract}

Some professions are classified as masculine, which means that the very practicing of the profession is proof of masculinity. Such is the case with the profession of police officer, whose job it is to crack down. Now that women have full access to police activities, it is no longer a question of encouraging women to enter the profession. Women's integration undergoes rather the in-depth examination of selection criteria for skills required by the profession so that these latter may line up with what we expect from the police nowadays.

Key words : police - discrimination sexism

$\begin{array}{lllllllll}R & E & C & H & E & R & C & H & E\end{array}$

\section{POLICIÈRES EN MARGE DE LA « VRAIE » POLICE}

\section{" MAMAN, EN DEHORS DE FAIRE PIPI DEBOUT, QUE FONT LES GARS QUE LES FILLES NE FONT PAS ? »}

ADAMS, 3 ans.

$$
\begin{aligned}
& \text { Line Beauchesne, Ph.D., } \\
& \text { professeure, Département de criminologie, } \\
& \text { Université d'Ottawa. }
\end{aligned}
$$

L'accès des femmes à l'entièreté des tâches policières dans les années 1970 est venu menacer ces lieux d'affirmation masculine, et ce, avec d'autant plus de force que cette affirmation est vécue avec peu d'extériorité en milieu policier. C'est que la structure des relations de travail dans cette profession est dominée par le concept de carrière intégrale, surtout en milieu non syndiqué comme à la GRC (Dowling et MacDonald, 1983; French et Béliveau, 1979 ; Reiner, 1992). Le concept de carrière intégrale signifie que s'engager dans cette profession est une vocation qui exclut toutes les autres et oriente même le mode de vie; on est policier jour et nuit. On ne travaille pas dans la police: on entre dans la police comme on entre en religion. On fait partie d'un groupe dont la solidarité et l'esprit de corps renforcent la distance avec la communauté (McLean, 1997 ; Monjardet, 1994; Reiner, 1992). Cette solidarité est si forte que l'on fait référence à la police comme à une entité avec des intentions et des caractéristiques personnelles à laquelle on voue une allégeance professionnelle (Fielding, 1994).
Cette solidarité professionnelle signifie également qu'il y aura une solidarité avec la culture masculine de travail. Dire qu'une culture de travail est sexuée signifie que les pratiques et les normes mêmes qui encadrent ces pratiques ne peuvent se comprendre que dans la distinction entre ce qui est féminin et ce qui est masculin (Acker, 1990).

Voyons comment les policières, qu'elles le veuillent ou non, viennent menacer cette solidarité envers la culture policière et se retrouvent en marge de celle-ci.

\section{UNE PROFESSION MASCULINE}

L'affirmation masculine basée sur des rapports de pouvoir sexistes signifie qu'être un homme repose plus sur la preuve que l'on n'est pas une femme que sur des affirmations de soi par des qualités positives. Dans ce type d'affirmation masculine, l'homme doit continuellement "prouver " aux autres hommes qu'il est un «vrai» homme, en montrant clairement qu'il n'a pas de traits féminins, d'où la phobie d'être accusé d'homosexuel.

Ce type d'affirmation masculine se bâtit dès l'enfance. Pas besoin de beaucoup de mots pour que le petit garçon ressente la déception de son père lorsqu'il pleure (comme une fille), lorsqu'il a peur (comme 
une fille), lorsqu'il est dépendant (comme une fille), etc. Pas besoin d'expliquer par des mots que les petits garçons se diminuent en adoptant des traits de personnalité dits féminins, lorsqu'on lui transmet que l'homosexualité est cette horreur d'un homme qui ne respecte plus l'identité masculine de puissance, de force et de virilité. En somme, les rapports de pouvoir sexistes sont inscrits dans le vocabulaire, les codes et certains rituels d'apprentissage de l'identité sexuelle (McCreary, 1994).

Et la petite fille qui affirme avec trop de vigueur son caractère et ses capacités physiques par rapport aux stéréotypes féminins ? C'est un garçon... manqué.

À l'âge adulte, la femme demeure la norme négative de cette affirmation d'identité masculine : être un homme, c'est ne pas être faible (comme une femme), peureux (comme une femme), trop émotif (comme une femme), etc. Et pour prouver aux autres hommes, juges de cette masculinité, que l'on est un «vrai » homme, il faut étaler sa force, sa bravoure, ses prouesses sexuelles, afficher son mépris des homosexuels, etc. (Kimmel, 1994 ; Hort et al., 1990 ; Rotundo, 1993).

C'est dans cette construction sexiste de l'identité sexuelle qu'il faut comprendre que dans une profession typée masculine, il y a les tâches "viriles », tâches valorisées, et les autres tâches, moins importantes pour définir la profession ; et la femme qui réussit à s'affirmer dans les tâches « viriles » vient menacer des lieux d'affirmation de la masculinité (Cockburn, 1991; Collinson, 1992) ${ }^{1}$.

Prenons par exemple le port de l'arme dans la police. Si l'homme qui porte une arme ajoute un trait à sa virilité (Dubé et Beauchesne, 1993), la femme qui porte une arme est "dénaturée » au regard des stéréotypes de féminité. Elle devra vraiment faire la preuve de sa compétence en la matière. Et si elle réussit à faire cette preuve, elle devient une menace.
On comprend mieux alors que la première génération de policières, dans les années 1970, ait fait l'objet de dizaines d'évaluations, mais pas pour l'ensemble des tâches de la profession. L'enjeu était de vérifier leurs capacités à exercer les fonctions «masculines» de la profession, même si cela ne constitue qu'une très petite partie des tâches policières : capacité de tir, capacité de conduire un véhicule à haute vitesse, de réagir à une situation d'agression, de faire des arrestations, etc. Plusieurs policières ont réagi à ces mesures discriminatoires, non seulement en montrant leur capacité à faire ces tâches, mais également en soulevant le caractère discriminatoire de certains critères de sélection qui n'avaient aucun lien avec les exigences de la profession. Les modifications qui suivirent demeurent perçues par de nombreux policiers comme une "diminution des exigences nécessaires à la profession », plutôt qu'une remise en question d'exigences dont l'inutilité dans l'accomplissement du travail policier a été démontrée (Breece et Garrett, 1977). Ce sont encore aujourd'hui des critères de sélection qui discriminent les femmes.

Par exemple, si la discrimination fondée sur la taille et le poids est, à toutes fins pratiques, abolie, la réussite des tests d'aptitudes physiques, encore fondés sur la force, demeure éliminatoire dans la majorité des corps de police. Cela indique la grande importance qu'on accorde encore aux tests d'aptitudes physiques pour entrer dans la profession. À quoi ressemblent-ils? Leur contenu est très variable selon les services. Les principaux modèles qui se dégagent sont les suivants:

1. Le test d'aptitudes physiques du Collège de police de l'Ontario (CPO) comprend une course d'un mille et demi, un test de souplesse avec redressement assis et étirement, un sprint de 100 verges, et une épreuve servant à déterminer le nombre maximum de redressements assis et de tractions sur les bras que le candidat peut effectuer en une minute. Certains services de police modifient ces épreuves pour les femmes.

2. L'exécution de tous ces tests, plus mesure de la capacité aérobique et anaérobique ainsi que du taux d'adiposité corporelle, exécution de tractions à la barre fixe et course d'agilité. On attribue à chaque composante du test une valeur, et le total de toutes les valeurs égale $100 \%$. Certains services de police modifient ces tests pour les femmes.

3. L'exécution des tests énumérés ci-dessus, en plus mesure de la force de la poignée (test du dynamomètre), mesure de la capacité aérobique, exécution de tractions à la barre fixe, tirage de poids et saut en hauteur. La note de passage pour ces tests d'aptitudes physiques varie aussi selon les services de police, entre $55 \%$ et $75 \%$.

4. Beaucoup de services de police utilisent le test d'aptitudes physiques du policier (POPAT) créé par le Justice Institute of British Columbia pour tous les candidats. Ce test inclut une course de mobilité et d'agilité d'un mille et quart, une activité de traction et de poussée, une activité modifiée d'appui facial avec projection des jambes vers l'arrière, station debout et saut latéral, et un port de poids $(45,5 \mathrm{~kg})$.

5. La GRC utilise le test d'aptitudes physiques essentielles (TAPE) comme instrument de sélection important pour tous les candidats. Le TAPE est un exercice de simulation qui comporte trois composantes, soit une course d'obstacles à six tours, un exercice de poussée et de traction, et le port d'un sac de $36 \mathrm{~kg}$ sur une distance

\section{Association des diplômés en études sur la mort (ADEM)

ADEM, Bureau des diplômés, local B-1105 Université du Québec à Montréal Case postale 8888 , succursale Centre-ville Montréal (Québec) H3C 3P8 Téléphone: (514) 987-3000, poste 3098 Télécopieur : (514) 987-8210 www.unites.uqam.ca/cem/adem

Adhésion, incluant 2 numéros de la revue Frontières

$\square$ Canada 40 \$ $\square$ Autres pays 50 \$

Prénom et nom:

Adresse:

Code postal:

Tél. rés.: ( ) Tél. travail:( ) 
de 15 mètres dans un délai donné.

Pour être reçus, les aspirants policiers doivent, à la fin de leur formation à l'école de la Gendarmerie, pouvoir transporter un sac de 45,5 kg (Walker, 1993, p. 32-33).

Plus de femmes que d'hommes échouent à ces tests. Pourtant, si ces tests sont si importants au point de constituer un facteur éliminatoire dans le processus de sélection des nouveaux policiers, comment se fait-il qu'aucun service de police n'impose une quelconque vérification de la forme physique des policiers comme condition de maintien à l'emploi ? En fait, la majorité des policiers et policières en service ne pourraient réussir ces tests, car ils correspondent au recrutement d'hommes jeunes et forts, et ils ont un effet discriminatoire à l'égard de presque toutes les personnes de plus de 30 ans (Byers, 1997). Ainsi les femmes plus matures, entre autres celles qui très tôt ont eu des enfants et qui décideraient par la suite d'entreprendre une carrière, ne peuvent avoir accès à la profession de policière. Plus encore, cette situation amène à s'interroger sur la pertinence même de ces tests comme critères d'entrée dans la profession (Peak, 1993 ; Roberg et Kuykendall, 1993 ; Stanfield, 1996; Townsey, 1982).

[...] le rationnel selon lequel la grosseur physique équivaut à la force qui signifie à son tour la compétence a été rejeté par des recherches empiriques.

Les femmes sont aussi capables d'assumer les fonctions reliées à la patrouille, sans pour cela avoir plus de chances d'être blessées lors des altercations, même si à l'évidence elles sont en général moins fortes que les hommes, ce qui ne signifie nullement une mauvaise condition physique.

(Lebeuf, 1996, p. 21-22)

Des tests d'aptitudes physiques différents pour les femmes plutôt que la remise en question de leur pertinence pour entrer dans la police maintiennent chez les policiers la perception de la nécessité de la force physique pour accomplir les fonctions policières et qu'à cet égard, les femmes représentent un risque dans la profession. Pourtant, ce critère de sélection éliminatoire aurait dû être aboli depuis longtemps, particulièrement dans le cadre du virage vers la police communautaire.

Plusieurs de ces exigences de force et d'agilité physiques sont plus reliées à l'image machiste de la police qu'aux exigences de l'emploi. Avec la reconnaissance croissante qu'une personne qui peut courir un mille mais n'a pas d'habiletés de communication peut ne pas être apte à être policier, on peut se poser des questions, à savoir si ces tests physiques sont vraiment nécessaires à l'emploi ou s'ils ne sont pas clairement discriminatoires à l'égard des femmes et des hommes plus petits. (Seagrave, 1997, p. 75-76. Notre traduction.)

La modification en profondeur des critères de sélection de manière à valoriser en priorité des compétences en communication permettrait non seulement aux candidats et candidates de faire valoir des expériences de travail qui ont mis en valeur ces qualités, mais accroîtrait également la perception de la compétence des femmes en matière d'intervention communautaire (Felkenes, Peretz et Schroedel, 1993).

De même, les différents véhicules de transmission des valeurs dans l'organisation policière indiquent bien la popularité qu'a toujours l'image paramilitaire de la police. L'arme, le badge et l'uniforme, de même que le grade à l'épaule, en témoignent. Ainsi, les résistances à l'intégration des femmes dans la police sont intimement liées à l'attachement à une culture policière où la force et l'autorité, le port de l'arme et l'arrestation constituent des symboles importants. Dans ce contexte, les femmes représentent le changement. Pourtant, les facteurs qui motivent les femmes à entrer dans la police ne diffèrent pas de ceux qui inspirent leurs collègues masculins (Charles, 1982; Christie 1992; Ermer, 1978 ; Jones, 1986 ; Lebeuf, 1996; Meagher et Yentes, 1986; Worden, 1993). De plus, comme les hommes, de nombreuses femmes ont choisi d'entrer dans la police parce que quelqu'un de la famille exerçait ce métier (Forcese, 1999; Jackson, 1992; Jones, 1986).

On comprend mieux, dès lors, que les femmes n'entrent pas dans la police avec l'intention, au départ, de modifier la culture masculine de travail; elles comptent pouvoir s'y intégrer en mettant l'énergie et le travail nécessaires. Elles désirent, par ce choix de carrière, acquérir la sécurité d'emploi et l'autonomie professionnelle (Fielding, 1994 ; Lasley, 1992). Elles croient, en majorité, que les femmes sont égales aux hommes et ont droit aux mêmes chances qu'eux d'obtenir un emploi dans la police. Mais elles acceptent, pour la plupart, les règles de la culture organisationnelle, qu'elles croient nécessaires à la fonction, prêtes à jauger à l'aune de leur mérite individuel leur succès ou échec dans la profession. Elles considèrent également naturel que le double rôle travail/famille soit " affaire de femmes». C'est à elles de faire en sorte, par une planification adéquate, que leurs affaires personnelles ne viennent pas entraver leur production au travail (Belknap, 1991; Horne, 1980 ; Jackson, 1997 ; Martin, 1980).
Comment se passe alors leur intégration professionnelle?

\section{FEMME OU POLICIÈRE}

Les policières n'ont pas simplement à faire la preuve qu'elles peuvent être de bonnes policières pour que tombent les préjugés sexistes à leur égard. Les études constatent que les croyances sont plus fortes que les faits lorsque les préjugés sont profonds. Et les préjugés sexistes sur les femmes au travail sont d'autant plus profonds qu'ils font écho à des préjugés sexistes hors travail, qui viennent en quelque sorte les « rationaliser», leur donner un fondement (Barber, 1992; Breakwell, 1990; Hoffman et Hurst, 1990). Comment se passe le maintien de ces préjugés? Pour maintenir des préjugés négatifs envers un groupe, les gens retiennent les comportements du groupe discriminé qui viennent les confirmer, même des petits détails, en négligeant la masse d'éléments qui les contredisent (Alcock et al., 1994 ; Hamilton et al., 1990).

Le jeu des stéréotypes sexistes au travail va alors rendre difficile pour une policière la tâche de préserver sa crédibilité à la fois comme policière et comme femme. Par exemple, les policières qui demandent de l'aide à leurs supérieurs dans les situations jugées à haut risque sont perçues comme compétentes en tant que femmes parce que l'on s'attend à cela d'elles, selon les stéréotypes, mais pas en tant que policières. Mais si elles ne demandent pas d'aide et vont «au-devant des coups », elles ne sont plus de «vraies » femmes (Grennan, 1993; Smith et DeWine, 1991). Cet exemple n'est pas choisi au hasard. Selon la plus grande enquête effectuée au Canada auprès des femmes policières, leur crédibilité professionnelle repose encore sur ce type d'attentes auprès de leurs collègues :

Il est intéressant de noter que, lorsqu'elles [les policières] ont été priées de citer un incident critique qui, à leur avis, avait joué un rôle déterminant dans leur acceptation par leurs collègues du sexe masculin à un point antérieur de leur carrière, les policières ont, sans exception, mentionné un incident de nature physique durant lequel elles s'étaient montrées capables de se défendre et (ou) de défendre leur partenaire. (Walker, 1993, p. 167)

Selon une enquête de Hunt (1992), il vaut mieux avoir fait preuve de trop d'agressivité et se faire dire de se calmer, ce qui confirme que l'on est une «vraie police» auprès de ses collègues, que d'avoir manqué d'agressivité là où il en fallait (selon eux), ce qui aurait confirmé que l'on n'était pas fait pour ce genre de travail. Cette situation comporte un risque pour les jeunes 
policières : si on perçoit qu'elles sont moins aptes à utiliser la «force nécessaire», elles seront encouragées plus souvent que les jeunes policiers à faire preuve d'autorité agressive pour prouver leur compétence professionnelle (Grennan, 1993). Ou encore, lorsque les policières refusent de se conformer à ce type de critère pour faire la preuve de leur professionnalisme, leur compétence professionnelle pourra être mise en doute et elles n'auront accès qu'à des fonctions et tâches dites féminines.

Possiblement à cause des obstacles auxquels font face les femmes à leur entrée dans la police, des recherches suggèrent que deux rôles professionnels différents peuvent se développer: la police-femme et la femme-police. La première tente de gagner l'approbation de ses collègues masculins en adhérant aux valeurs et aux normes de la police traditionnelle, avec le contrôle du crime comme première orientation; la seconde tente d'accomplir ses fonctions «d'une manière stéréotypée féminine ». Elle fait peu d'arrestations, évite les incidents de nature physique, et s'efforce de demeurer une "vraie femme». (Roberg et Kuykendall, 1993, p. 402. Notre traduction.)

Le choix par les policières de s'affirmer principalement comme femmes-polices ou polices-femmes peut créer des tensions entre ces deux groupes, chacun considérant que l'autre groupe nuit à son affirmation sexuelle ou à son identité professionnelle (Brewer, 1991 ; Epstein, 1992 ; Jones, 1986; Martin, 1987). La présence croissante de modèles féminins ces dernières années atténue cette dualité, selon les études, en donnant raison aux femmes de croire qu'elles peuvent se distinguer sans se mettre en péril, soit comme femmes, soit comme professionnelles (Horne, 1980; Jurik, 1988; Lips et Colwill, 1993; van Nostrand, 1993). C'est la reconnaissance en tant que policière.

\section{FEMME ET POLICIÈRE}

Que font plusieurs policières pour être perçues en tant que femmes professionnellement compétentes?

Dans une enquête auprès des gardiennes de prison, Jurik (1988) a identifié cinq stratégies que l'on peut aisément transposer chez les policières.

La première est de conserver une image professionnelle en évitant la camaraderie et en collant aux règles que l'on connaît, bien sûr, par cœur. Cette stratégie permet à la policière d'être moins vulnérable aux tentations de manipulation quand elle arrive dans le milieu, mais à court terme seule- ment. Faire durer cette stratégie entraîne la perception que l'on est incapable d'utiliser son jugement pour se distancier de ces règles quand c'est nécessaire. Et si l'on gagne en pouvoir en persistant dans cette stratégie, les rapports avec les subalternes peuvent devenir extrêmement difficiles.

La seconde stratégie est de faire ressortir son expérience unique comme femme. Cela évite ainsi, politiquement, d'entrer en compétition avec les collègues masculins tout en faisant preuve de compétence. La limite de cette stratégie est de cloisonner les femmes dans des tâches spécifiques, ce qui rend difficiles les transferts dans d'autres secteurs ou encore limite les promotions parce que ces policières sont trop spécialisées. Également, cela entretient l'image de la femme incapable d'effectuer les tâches typées masculines.

La troisième stratégie est de s'identifier à une équipe, particulièrement si l'on a fait un bon coup, pour éviter d'être mise en évidence au détriment des hommes. Le problème est que même si le travail d'équipe est mis en valeur par les gestionnaires dans la police, en pratique, on ne donne pas des promotions à des équipes, mais à des individus. Cette stratégie cache les succès des policières.

La quatrième stratégie est l'humour. Cette stratégie, jugée importante par l'ensemble des policières selon les enquêtes (TNT, 1988; Martin, 1987; Walker, 1993), est relativement profitable pour échanger avec le public et les collègues. Mais c'est une arme à double tranchant; cela peut également signifier, dans un contexte de discrimination sexiste, l'acceptation du harcèlement sexuel ou sexiste.

La cinquième stratégie consiste à rechercher le protectorat d'un mentor. Le problème est que si ce protectorat est trop affiché, on soupçonnera immédiatement qu'il s'accompagne de relations sexuelles; si la femme obtient une promotion, la légitimité de sa promotion sera mise en doute. S'il y a brouille avec le mentor, on pensera à la rupture amoureuse, etc. S'il s'agit d'une mentor, la solidarité féminine sera l'explication des promotions, indépendamment des compétences.

En somme, comme elles sont en minorité et que les processus organisationnels pour les intégrer ne sont pas encore efficaces ou suffisants, les policières risquent, pour répondre aux attentes contradictoires du milieu à leur égard, de glisser dans des rôles-pièges qui minent leur crédibilité en tant que policières ou en tant que femmes, ou encore d'adopter des stratégies professionnelles qui nuisent à leur avancement. Est-ce que l'augmentation du nombre de policières viendra faire disparaître ces problèmes?

\section{LE MYTHE DU NOMBRE MAGIQUE}

Les organisations policières doivent abandonner le mythe confortable voulant qu'au fur et à mesure que les policières seront plus nombreuses, tant à la base qu'aux postes décisionnels, les éléments de la profession qui créent des obstacles à leur intégration disparaîtront d'eux-mêmes. D'une part, il ne revient pas aux femmes de changer la culture policière et, d'autre part, il ne faut pas compter que plus de policières signifie plus d'agentes de changement de cette culture, les motivations des hommes et des femmes à entrer dans la profession étant identiques.

Cela ne signifie pas que les organisations policières ne doivent pas travailler à accroître activement et considérablement le nombre de femmes dans la police. C'est d'abord et avant tout une question de justice sociale (Bégin, 1998). De plus, une étude récente de Lortie-Lussier (2001) indique que lorsqu'il existe une proportion de plus de $20 \%$ de femmes dans une profession, les évaluations positives de leurs compétences et l'acceptation de leur présence s'accroissent considérablement chez les hommes. Elle note toutefois qu'en ce qui concerne les femmes aux postes de gestion, les résultats sont plus variables. Cela tiendrait entre autres au fait qu'à ce niveau hiérarchique, plusieurs des postes sont occupés par des hommes qui n'ont pas fait leurs études en compagnie de femmes et / ou qui n'ont pas eu d'expérience de travail avec des femmes, contrairement aux plus jeunes. Les changements d'attitudes sont alors plus difficiles chez nombre d'entre eux.

Toutefois, il ne faut pas confondre ces changements d'attitude à l'égard des femmes avec l'abolition des obstacles à leur intégration. Cette confusion amène aisément les organisations à attendre un nombre magique de femmes dans la profession et à restreindre leur action à quelques gestes publics pour prouver qu'elles font quelque chose, plutôt que de travailler activement à changer les obstacles de fond qui touchent à la culture organisationnelle de la profession.

Ce rôle proactif pour soutenir les policières et assumer les nouvelles problématiques suscitées par leur présence signifie, principalement :

- l'examen des pratiques de travail pour distinguer celles qui relèvent des

nécessités de la fonction, de celles qui relèvent d'une culture masculine et qui constituent une discrimination inutile à l'égard des femmes ;

- l'examen des pratiques discriminatoires dans les évaluations et les promotions ;

- la mise en place de politiques, de stratégies de prévention et de services en matière de harcèlement sexiste et sexuel; 
- la prise en compte des couples policiers au travail;

- la réduction des difficultés de conciliation du travail et de la famille.

Ces actions ne sont pas que des pratiques mesurables quantitativement et de simples procédures formelles, même importantes, ne suffisent pas à résoudre les problèmes. Si les organisations policières limitent leur rôle à cela sans tenir compte des résistances socio-émotives en jeu dans la remise en question de la culture professionnelle, «de telles mesures risquent de susciter l'apparition de subtiles opérations de sabotage ou de créer, au sein d'une organisation, des tensions qui pourraient s'avérer coûteuses pour tous et à tous les niveaux [...] » (Cormier, 1990, p. 462)

Les policières en savent quelque chose, elles qui vivent les ressacs des collègues à cause de mesures spéciales pour les accommoder, plutôt que de changements en profondeur dans la culture organisationnelle. Pour ces raisons, plusieurs en sont venues à craindre et même à détester les programmes d'équité qui entraînent une diminution de la crédibilité à l'égard de leurs compétences et qui suscitent même de l'hostilité à leur présence. Les organisations policières doivent cesser d'attendre qu'un nombre magique de policières arrange tout. Elles doivent veiller à ce que l'implantation des programmes d'équité soit accompagnée de stratégies qui touchent à la transformation des valeurs et des attitudes responsables de ces ressacs.

Au cœur de ces changements, il y a la remise en question par les organisations policières de l'image même de la police telle qu'elle s'est développée depuis le début du siècle, où la fonction de répression s'est inscrite dans une symbolique militaire de combat et une gestion paramilitaire. Dans ce contexte, l'affirmation masculine traditionnelle est venue s'inscrire dans la culture et les pratiques, répondant aux rapports sociaux de sexe. L'importance encore aujourd'hui de la force physique dans les critères de sélection et le caractère paramilitaire des structures de gestion témoignent de la persistance de ce passé lourd de conséquences pour les policières.

Le discours qui fait reposer les changements de cette culture professionnelle sur les aptitudes «naturelles» qu'auraient les femmes vers d'autres formes d'intervention plus conciliatoires et d'autres formes de gestion plus participatives, nie la responsabilité qu'ont les organisations policières d'effectuer des changements en profondeur de la culture policière. Il accentue également le ressentiment à l'égard des policières en opérant certains des changements dans cette direction à travers les programmes d'équité comme, par exemple, la modifica- tion de normes discriminatoires de sélection et de promotion qui ne correspondent pas aux exigences de la fonction. Si certaines normes ne correspondent pas aux exigences de la fonction, elles sont discriminatoires pour tout le monde, hommes et femmes. À cet égard, elles doivent s'inscrire dans une remise en question des exigences mêmes de la profession, et non à l'intérieur des programmes d'équité. Les maintenir à l'intérieur de ces programmes, c'est maintenir une culture professionnelle masculine, où la compétence des femmes est moins reconnue.

Ainsi, il ne s'agit pas de «féminiser » la profession en remettant en question la «vraie police». L'intégration des femmes passera par l'examen en profondeur des critères de sélection et des compétences exigées par la profession afin que ces critères et compétences correspondent à ce que nous attendons de la police aujourd'hui. Comme on peut le voir, l'intégration des femmes, à cet égard, s'inscrit dans les débats beaucoup plus larges qui ont lieu à l'heure actuelle sur le rôle de la police.

Ces éléments renvoient aussi à des problématiques sociales plus globales: les rapports de pouvoir entre les sexes dans la société, le support étatique à la famille, les critères organisationnels du profil idéal de carrière, etc.

Que veulent les policières? À elles de le définir. Mais pour les entendre et soutenir leurs revendications, les gestionnaires, de même que les gouvernements, devront jouer activement leur rôle dans la transformation de la culture policière.

Sortir les policières de la marge, c'est faire une réflexion en profondeur sur le rôle de la police dans nos sociétés.

\section{BIBLIOGRAPHIE}

ACKER, J. (1990). «Hierarchies, jobs, bodies: A theory of gendered organizations ", Gender \& Society, vol. 4, n 2, p. 139-158.

ALCOCK, J.E., D.W. CARMENT et S.W. SADARA (1994 - 3e éd.). A Textbook of Social Psychology, Ontario, Prentice Hall Canada Inc., chap. 6.

BARBER, P.G. (1992). "Conflicting loyalties. Gender, class and equity politics in working class culture.», Canadian Woman Studies, vol. 12, p. 80-83.

BEAUCHESNE, L. (2001). Policière: une profession masculine, Montréal, Éditions du Méridien.

BEAUCHESNE, L. (1999). «Les recherches en Amérique du Nord sur l'entrée des femmes dans la police: les difficultés d'intégration dans une culture organisationnelle masculine », Déviance et Société, vol. 23, n 3, p. 341-362.

BÉGIN, M. (1998). Towards a Critical Mass: Women in Politics, JP Naik Memorial Lecture, New Delhi, Centre for Women's Development Studies.
BELKNAP, J. (1991). «Women in conflict: An analysis of women correctional officers », Women \& Criminal Justice, vol. 2, $\mathrm{n}^{\circ} 2$, p. 89-115.

BERG, B.L. (1992). Law Enforcement, Boston, Allyn and Bacon, chap. 9-10.

BREAKWELL, G.M. (1990). "Social beliefs about gender differences », dans C. FRASER et G. GASKELL (dir.), The Social Psychological Study of Widespread Beliefs, Oxford, Clarendon Press, p. 210-225.

BREECE, C.M. et G.R. GARRETT (1977). "Women in policing: Changing perspectives on the role» dans J.E. SCOTT et S. DINITZ (dir.), Criminal Justice Planning, New York, Praeger Publishers, chap. 1.

BREWER, J.D. (1991). «Hercules, Hippolyte and the Amazons - or policewomen in the RUC », British Journal of Sociology, vol. 42, $\mathrm{n}^{\circ} 2$, p. 231-247.

BYERS, E.S. (1997). La situation des femmes et des minorités visibles dans la police au Nouveau-Brunswick, Rapport préparé pour la Division des services de police du ministère du Solliciteur général du NouveauBrunswick, 31 octobre.

CHARLES, M.T. (1982). "Women in policing: The physical aspect», Journal of Police Science and Administration, vol. 10, $\mathrm{n}^{\circ} 2$, p. 194-205.

CHRISTIE, G. (1992). Choosing a Career in Policing: Service, Law an Order and the Effects of Gender, Polycopié.

COCKBURN, C. (1991). In the Way of Women: Men's Resistance to Sex Equality in Organizations, Ithaca, ILR Press.

COLLINSON, D.L. (1992). Managing the Shopfloor: Subjectivity, Masculinity and Workplace Culture, New York, Walter de Gruyter.

CORMIER, S. (1990). "Les interventions auprès des femmes. Formes actuelles et perspectives d'avenir », dans R. TESSIER et Y. TELLIER (dir.), Méthodes d'intervention, développement organisationnel, Québec, PUQ, chap. 14.

DOWLING, J.B. et V.N. MacDONALD (1983). Les réalités sociales du travail policier: essais sur la théorie de la légitimation, Ottawa, Collège canadien de police.

DUBÉ, Y. et L. BEAUCHESNE (1993). Désarmer la police? Un débat qui n'a pas eu lieu, Montréal, Éditions du Méridien.

EPSTEIN, C.F. (1992). "Tinkerbells and pinups: The construction and reconstruction of gender boundaries at work», dans Michèle LAMONT et Marcel FOURNIER (dir.), Cultivating Differences, Chicago, The University of Chicago Press, chap. 10.

ERMER, V.B. (1978). "Recruitment of female police officers in New York », Journal of Criminal Justice, vol. 6, n 3, p. 233-245.

FELKENES, G.T. et J.R. SCHROEDEL (1993). "A case study of minority women in policing», Women \& Criminal Justice, vol.4, no 2, p. 65-89.

FELKENES, G.T., P. PERETZ et J.R. SCHROEDEL (1993). "An analysis of the 
mandatory hiring of females: The Los Angeles Police Department experience ", Women \& Criminal Justice, vol.4, no 2, p. 31-63.

FIELDING, N.G. (1994). "Cop canteen culture", dans T. NEWBURN et E.A. STANKO (dir.), Just Boys Doing Business?, Londres, Routledge, chap. 3.

FORCESE, D. (1999, 2e éd.). Policing Canadian Society, Ontario, Prentice-Hall Canada Inc.

FRENCH, R. et A. BÉLIVEAU (1979). La GRC et la gestion de la sécurité nationale, Toronto, Institut de recherches politiques.

GRENNAN, S. (1993). "A perspective on women in policing», dans R. MURASKIN et T. ALLEMAN (dir.), It's a Crime, Women and Justice, New Jersey, Prentice Hall, chap. 9.

HAMILTON, D.L., S.J. SHERMAN et C.M. RUVOLO (1990). "Stereotype-based expectancies: Effects on information processing and social behavior", Journal of Social Issues, vol. 46, $\mathrm{n}^{\circ} 2$, p. 35-60.

HOFFMAN, C. et N. HURST (1990). «Gender stereotypes: Perception or rationalization? », Journal of Personality and Social Psychology, vol. 58, n 2, p. 197-208.

HORNE, P. (1980). Women in Law Enforcement, Illinois, Charles C. Thomas Publisher.

HORT, B.E., B.I. FAGOT et M.D. LEINBACH (1990). "Are people's notions of maleness more stereotypically framed than their notions of femaleness? », Sex Roles, vol. 23, $\mathrm{n}^{\text {os }} 3-4$, p. 197-212.

HUNT, J. (1992 - 4e éd.). "New cops on the street: Learning normal force», dans C. CLARK et H. ROBBOY (dir.), Social Interaction, Readings in Sociology, New York, St. Martin's Press p. 86-96.

HUNT, J. (1990). "The logic of sexism among police », Women \& Criminal Justice, vol. $1, \mathrm{n}^{\circ} 2$, p. 3-30.

INSTITUT DE POLICE DU QUÉBEC, (1999). S'unir pour grandir ensemble, Actes du Colloque sur la femme policière, Nicolet (Québec), Institut de police du Québec.

JACKSON, L.A. (1997). Physical Appearance and Gender State, University of New York Press, chap. 4.

JACKSON, R.L. (1992). Employment Equity and Ontario Police, Queen's University, Industrial Relations Centre.

JONES, S. (1986). Policewomen and Equality, Hampshire, The Macmillan Press Ltd.

JURIK, N.C. (1988). «Striking a balance: Female correctional officers, gender role stereotypes, and male prisons ", Sociological Inquiry, vol. 58, no 3, p. 291-305.

JURIK, N.C. (1985). «An officer and a lady: Organizational barriers to women working as correctional officers in men's prisons ", Social Problems, vol. 32, no 4, p. 375-388.

KIMMEL, M.S. (1994). "Masculinity as homophobia. Fear, shame, and silence in the construction of gender identity ", dans H. BROD et M. KAUFMAN (dir.), Theorizing Masculinities, Londres, Sage Publications, chap. 7.
KOLB, D.M. (1992). «Women's work. Peacemaking in organizations", dans D.M. KOLB et J.M. BARTUNEK (dir.), Hidden Conflict in Organizations, Londres, Sage Publications, chap. 3.

LASLEY, J. (1992). "Fulfilling the mandates of the law: The consent decrees and compliance ", dans G.T. FELKENES et P.C. UNSINGER (dir.), Diversity, Affirmative Action and Law Enforcement, Illinois, Charles C. Thomas Publisher, p. 137-167.

LEBEUF, M.E. (1996). Trois décennies de femmes dans la police. Une bibliographie commentée, Ottawa, Collège canadien de police.

LIPS, H. et N. COLWILL (1993, 2 é éd.). "Issues in the workplace", dans H.M. LIPS (dir.), Sex and Gender, An Introduction, Londres, Mayfield Publishing Company, chap. 13.

LORTIE-LUSSIER, M. (2001). «The proportion of women managers: Where is the critical mass? », Journal of Applied Social Psychology.

MARTIN, J. (1992). "The suppression of gender conflict in organizations » dans D.M. KOLB et J.M. BARTUNED (dir.), Hidden Conflict in Organizations, Londres, Sage Publications, chap. 7.

MARTIN, P.Y. (1992). "Gender, interaction, and inequality in organizations », dans C.L. RIDGEWAY (dir.), Gender, Interaction and Inequality, New York, Springer-Verlag, p. 208-231.

MARTIN, S.E. (1991). "The effectiveness of affirmative action: The case of women in policing », Justice Quarterly, vol. 8, $\mathrm{n}^{\circ} 4$, p. 489-504.

MARTIN, S.E. (1990). On the Move: The Status of Women in Policing, Washington, Police Foundation.

MARTIN, S.E. (1987). "Sexual politics in the workplace: The interactional world of policewomen", Women and Symbolic Interaction, USA, Allen \& Unwin Inc., chap. 17.

MARTIN, S.E. (1980). Breaking and Entering, Policewomen on Patrol, Berkeley, University of California Press.

McCREARY, D.R. (1994). "The male role and avoiding feminity", Sex Roles, vol. 31, $\mathrm{n}^{\text {os }}$ 9-10, p. 517-529.

McLEAN, J. (1997a) «Les effets de la culture policière sur l'identité sociale », dans M.E. LEBEUF et J. McLEAN (dir.), Les femmes dans la police au Canada: Les années 2000 et après - les défis, Actes du séminaire tenu au Collège canadien de police du 20 au 23 mai 1997, Ottawa, Collège canadien de police, p. 63-70.

McLEAN, J. (1997b) «Perspectives d'avenir des femmes dans la police au Canada», Notes finales idem, p. 219-226.

MEAGHER, M.S. et N.A. YENTES (1986). "Choosing a career in policing: A comparison of male and female perceptions ", Journal of Police Science and Administration, vol. $14, \mathrm{n}^{\circ} 4$, p. 320-327.
MONJARDET, D. (1994). «La culture professionnelle des policiers ", Revue française de sociologie, vol. XXXV, $\mathrm{n}^{\circ}$ 393-411.

PEAK, K.J. (1993). Policing America, New Jersey, Prentice Hall.

REINER, R. (1992, 2 ${ }^{\mathrm{e}}$ éd.). The Politics of the Police, Toronto, University of Toronto Press.

ROBERG, R.R. et J. KUYKENDALL (1993). Police \& Society, California, Wadsworth Publishing Co., chap. 12.

ROTUNDO, E. A. (1993). American Manhood: Transformations in Masculinity from the Revolution to the Modern Era, New York, Basic.

SEAGRAVE, J. (1997). Introduction to Policing in Canada, Ontario, Prentice Hall Canada Inc.

SEAGRAVE, K. (1995). Police Women: A History, Caroline du Nord, McFarland \& Company Inc.

SMITH, G.L. et S. DeWINE (1991). «Perceptions of subordinates and requests for support. Are males and females perceived differently when seeking help ? ", Group \& Organization Studies, vol.16, n ${ }^{\circ} 4$, p. 408427.

STANSFIELD, R.T. (1996). Issues in Policing, A Canadian Perspective, Toronto, Thompson Educational Publishing Inc.

TOWNSEY, R.D. (1982). "Female patrol officers: A review of the physical capability issue» dans B.R. PRICE et N.J. SOKOLOFF (dir.), The Criminal Justice System and Women, New York, Clark Boardman Company Ltd., p. 413-425.

TRAVAIL NON TRADITIONNEL INC. (TNT) (1988). Rapport sur l'intégration des policières du SPCUM, Polycopié.

Van NOSTRAND (1993). Gender-Responsible Leadership, New York, Sage Publications, chap. 4.

WALKER, S.G. (1993). La situation des femmes dans la police au Canada: l993, Ottawa, Solliciteur général du Canada.

WORDEN, A.P. (1993). «The attitudes of women and men in policing: Testing conventional and contemporary wisdom», Criminology, vol. 31, n 2 , p. 203-241.

\section{Sites Internet}

INTERNATIONAL ASSOCIATION OF WOMEN POLICE

http://www.iawp.org/

EUROPEAN NETWORK

OF POLICEWOMEN

http://www.enp.nl/nnetwofpwomen.htm

NATIONAL CENTER

FOR WOMEN AND POLICING

http ://www.feminist.org/wi/com/police

\section{Note}

1. Et, il va sans dire, les policiers homosexuels cachent le plus possible leur orientation sexuelle pour éviter la discrimination. 